\title{
CERTIFICAÇÃO DE PRODUTOS FLORESTAIS NÃO MADEIREIROS NA PERSPECTIVA MERCADOLÓGICA DE ASSOCIAÇÕES EXTRATIVISTAS NO ESTADO DO ACRE
}

\author{
A. M. IMPERADOR ${ }^{1}$ e L. H. O. WADT ${ }^{2}$ \\ ${ }^{1}$ Universidade Federal de Alfenas - campus Avançado de Poços de Caldas. \\ ${ }^{2}$ Embrapa Acre.
}

Artigo submetido em novembro/2012 e aceito em fevereiro/2014

\section{RESUMO}

A Certificação concedida pelo Conselho de Manejo Florestal é um instrumento de gestão para o uso sustentável dos recursos florestais, considerando aspectos sociais, econômicos e ambientais. Dentre suas vantagens está a possibilidade de conquistar novos mercados e agregar valor ao produto. Diante da experiência das Associações dos Seringueiros Porto Dias e dos Moradores e Agroextrativistas do Remanso de Capixaba, ambas do Estado do Acre, este estudo avaliou a percepção de seus manejadores em relação ao mercado para Produtos Florestais Não Madeireiros certificados através de en-trevistas estruturadas. Nesse universo que representou $100 \%$ das famílias certificadas nas duas Associações, $53 \%$ dos entrevistados consideraram as questões relativas ao mercado como maior dificuldade encontrada para man-ter a certificação enquanto que 59\% apontaram que melhoria das condições de comercialização de seus produtos como o principal entrave para a manutenção do processo. Verificou-se que agregar valor e comercia-lizar o produto com vantagem competitiva são condições essenciais para a viabilidade da Certificação Florestal Comunitária como incentivo ao manejo sustentável dos recursos florestais na Amazônia.

PALAVRAS-CHAVE: Certificação Florestal, Amazônia, produtos não madeireiros.

\section{CERTIFICATION OF NON-TIMBER FOREST PRODUCTS IN THE MERCHANTABILITY VIEW OF EXTRACTIVE ASSOCIATIONS IN THE STATE OF ACRE}

\begin{abstract}
The certification granted by the Forest Stewardship Council is a management tool for the sustainable use of forest resources, considering social, economic and environmental aspects. The possible advantages include the ability to win new markets and add value to the products. The efforts in promoting the community certification for the development of rural populations as well as for forest conservation present many challenges, such as the lack of scientific studies that address traditional knowledge. Given the experience of the Associações dos Seringueiros Porto Dias and Associação dos Moradores e Agroextrativistas do Remanso de Capixaba, both from the State of Acre, Brazil, the study herein assesses the manager's perception regarding the
\end{abstract}

market for Certified Non-Timber Forest Products, through structured interviews. In this sphere representing $100 \%$ of the certified families that were surveyed in both associations, it was found that $53 \%$ of the respondents considered the issues concerning the market as the most difficult aspect encountered to maintain certification, while $59 \%$ pointed to improvement in the marketing of its products as the main bottleneck to have access to the market. It was found that adding value and marketing the product with a competitive advantage are essential conditions for the Community Forest Certification as an incentive for the sustainable management of resources in the Amazon forest.

KEYWORDS: Forest Certification, The Amazon Forest, non-timber products. 


\section{INTRODUÇÃO}

O uso sustentável tem importante papel na conservação dos recursos naturais na floresta amazônica (BRECHIN et al, 2002; NEPSTAD et al, 2002; HEYWOOD \& IRIONDO, 2003; WADT et al, 2008). O conceito de sustentabilidade foi consolidado através da relação entre as populações humanas e o meio ambiente (LIMA \& POZZOBON, 2005).

Uma estratégia bastante utilizada para conservar os recursos naturais tem sido a promoção do Manejo Florestal Comunitário (AMARAL \& AMARAL NETO, 2005). Neste sentido, a Certificação Florestal concedida pelo Conselho de Manejo Florestal (FSC) é uma ferramenta de incentivo a esta prática, através de mecanismo de controle da origem de produtos florestais.

Até o ano de 2009, apenas 3,73\% das Florestas Certificadas pelo FSC eram manejadas por comunidades, e embora este número seja crescente, ainda é insipiente frente às certificações com base empresarial. Com o objetivo de incentivar e viabilizar a Certificação Florestal Comunitária, o FSC incorporou no sistema o Padrão de Manejo Florestal de Pequena Escala e Baixa Intensidade, com custo de avaliações reduzidas e avaliações mais práticas (WENBAN-SMITH et al, 2001; HIGMAN, NUSSBAUM, 2002; ROBINSON, BROWN, 2002; SHANLEY et al, 2005). A Certificação de Produtos Florestais não Madeireiros (PFNM's), que compreendem os frutos comestíveis, óleos, látex, sementes, cipós, resinas, entre outros, tem sido incentivado nesta década, (PINTO et al, 2008). Estima-se que os PFNM's respondem por até $25 \%$ da renda para cerca de um bilhão de pessoas no mundo todo (MOLNAR et al, 2004) e que cerca de $80 \%$ da população mundial depende de medicamentos tradicionais de componentes fitoterápicos (FARNSWORTH et al, 1985). Estes indicadores fatos estimularam o investimento em estudos com diferentes focos e perspectivas disciplinares como mercado, antropologia e estudos biológicos voltados para o manejo sustentável de PFNM's (WADT et al, 2003).

Em relação aos benefícios ambientais, os PFNM's são considerados uma alternativa a conservação de florestas tropicais, por serem menos destrutivos do ponto de vista ecológico do que a exploração madeireira (MYERS et al, 2000) desde que haja alternativas de renda para extração de recursos sazonais (ESCOBAL \& ALDANA, 2003) e que as comunidades consigam comercializar seu produto com valor agregado, dando viabilidade ao processo (SHANLEY et at., 2005). Vale ressaltar que benefícios do mercado são considerados como principais motivações para quem busca a certificação (THORNBER, MARKOPOULOS, 2001; QUEVEDO, 2006; HUMPHRIES, KAINER, 2006).

Todavia, os PFNM's compõem um dos grupos de produtos mais difíceis de certificar, especialmente devido a grande variedade de produtos e a complexidade da cadeia de custódia, (JONES et al, 2002; PIERCE \& LAIRD, 2003).

Neste contexto, as instituições de pesquisa têm discutido sobre as oportunidades e desafios para se implantar e manter a certificação nas comunidades, entretanto poucos estudos têm questionado a percepção dos manejadores (HUMPHRIES \&KAINER, 2006).

É fato que pesquisadores devem utilizar os conhecimentos tradicionais para buscar o desenvolvimento sustentável para sistemas florestais (ALCORN, 1989) e que desenvolvimento de padrões de certificação requer um processo consultivo, com a participação efetiva de representantes do governo, ONGs, pesquisadores e grupos comunitários (LIMA et al, 2009). 
Esta pesquisa é parte integrante do Projeto KAMUKAIA coordenado pela Embrapa, cujo objetivo é suprir demandas de pesquisa para o uso sustentável de Produtos Florestais não Madeireiros na Amazônia e apresenta a percepção de duas Associações de produtores extrativistas do estado do Acre sobre o mercado de produtos não madeireiros certificados, apontando as principais dificuldades enfrentadas pelos manejadores para manter a certificação e suas sugestões de mudanças para viabilizar a manutenção do processo.

\section{MATERIAL E MÉTODOS}

\section{1 Área de Estudo}

Esta pesquisa focou duas comunidades situadas no estado do Acre, na Amazônia Ocidental Brasileira; sendo elas a Associação Seringueira Porto Dias (ASPD), em área do Projeto Agroextrativista Porto Dias, e a Associação de Moradores e Agroextrativistas do Remanso de Capixaba (AMARCA), em área do Projeto Agroextrativista São Luiz do Remanso.

O Projeto Agroextrativista Porto Dias - PAE Porto Dias foi criado através do Decreto no 95.577, de 23 de dezembro de 1987. Trata-se da última área de floresta natural do município de Acrelândia, cerca de $140 \mathrm{~km}$ da capital do estado do Acre, Rio Branco. Apresenta uma área de 22.145,00 ha, onde atualmente vivem aproximadamente 105 famílias cadastradas junto ao INCRA - Instituto Nacional de Colonização e Reforma Agrária - uma Autarquia Federal. A Comunidade está organizada em Associações, sendo que a Associação Seringueira Porto Dias (ASPD) foi certificada para a exploração de madeira florestal no ano de 2002, sendo solicitada em 2003 a certificação para a produção do óleo de copaíba, por meio de um adendo no escopo da certificação, sendo em 2004, o primeiro caso de manejo florestal comunitário do Brasil a receber a certificação FSC para um PFNM (SMARTWOOD \&IMAFLORA, 2005).

O Projeto Agroextrativista São Luiz do Remanso (PAE São Luiz Remanso) foi criado através da Portaria do INCRA $n^{\circ}$. 627 de 30 de julho de 1987 e suas terras são concedidas sob regularização fundiária de forma coletiva, através de Contrato de Direito Real de Uso, firmado entre o INCRA e a Associação dos Moradores do PAE Remanso do Capixaba e do Plano de Utilização que estabelece diretrizes de organização e conduta da comunidade. Esta comunidade ocupa uma área de 39.570 hectares, compreendendo terras dos municípios de Capixaba e Rio Branco (SMARTWOOD \& IMAFLORA, 2006). O PAE São Luiz do Remanso está organizado em Associações, sendo que a AMARCA, Associação de Moradores e Agroextrativistas do Remanso de Capixaba, foi criada em 1994 com o objetivo de captar recursos para desenvolvimento das atividades produtivas e contribuir com a regulamentação fundiária dos assentados. No ano de 2003, a AMARCA solicitou auditoria completa de "Certificação Florestal e de Cadeia de Custódia", para os produtos madeira, jarina e óleo de copaíba.

A Tabela 1 apresenta algumas características das associações pesquisadas.

Tabela 1: Características sociais e da certificação florestal das associações alvo da pesquisa.

\begin{tabular}{ccc}
\hline ASSOCIAÇÃO & ASPD & AMARCA \\
\hline $\mathrm{N}^{\circ}$ de associados (2008) & 20 & 81 \\
\hline $\begin{array}{c}\mathrm{N}^{\circ} \text { de famílias certificadas com } \\
\text { PFNM's ativas (2008) }\end{array}$ & 7 & 10 \\
\hline
\end{tabular}




\begin{tabular}{|c|c|c|}
\hline Ano de certificação & $\begin{array}{c}2002 \text { - Madeira } \\
2004 \text { - Inclusão de PFNM's }\end{array}$ & 2004 - Mista \\
\hline Tipo de certificação & Mista & Mista \\
\hline Ano de certificação dos PFNM's & 2004 & 2004 \\
\hline PFNM certificado & Copaíba (Copaifera sp) & $\begin{array}{l}\text { Jarina (Phytelephas sp) } \\
\text { Copaíba (Copaifera sp) }\end{array}$ \\
\hline Certificadora & Imaflora & Imaflora \\
\hline Padrão de certificação utilizado & $\begin{array}{c}\text { Manejo Florestal de Terra Firme } \\
\text { para Amazônia Brasileira }\end{array}$ & $\begin{array}{c}\text { Manejo Florestal de Terra Firme } \\
\text { para Amazônia Brasileira }\end{array}$ \\
\hline Tipo de operação & $\begin{array}{l}\text { Manejo florestal comunitário de } \\
\text { uso múltiplo }\end{array}$ & $\begin{array}{l}\text { Manejo florestal comunitário de } \\
\text { uso múltiplo }\end{array}$ \\
\hline Instituição de apoio & $\begin{array}{c}\text { CTA - Centro dos Trabalhadores } \\
\text { da Amazônia }\end{array}$ & $\begin{array}{c}\text { CTA - Centro dos Trabalhadores } \\
\text { da Amazônia }\end{array}$ \\
\hline Situação atual (2008/2009) & Ativa & Ativa \\
\hline
\end{tabular}

Conforme documento oficial das auditorias realizadas pela ONG IMAFLORA (SMARTWOOD \& IMAFLORA, 2005, SMARTWOOD \& IMAFLORA, 2006), as Associações ASPD e AMARCA apresentam como principais fontes de geração de renda o manejo florestal de uso múltiplo, que inclui a exploração de Produtos Florestais Madeireiros e não Madeireiros, a agricultura de subsistência e a criação animais.

Embora a extração madeireira tenha certamente a maior participação na renda das famílias, a extração de PFNM's vem ganhando força devido a demanda de mercado por alguns produtos como a castanha do Brasil, o óleo de cobaíba para a indústria de fármacos e a jarina para a confecção de biojóias.

\subsection{Coleta de Dados}

Os dados foram coletados por meio de entrevista estruturada com perguntas do tipo aberta, semiabertas e fechadas (SILVA, 2005).

As entrevistas foram realizadas de janeiro a dezembro de 2008, na Associação dos Seringueiros de Porto Dias foram entrevistadas sete famílias $(n=7)$ e na Associação dos Moradores Agroextrativistas de Remanso do Capixaba dez $(n=10)$, sendo contempladas $100 \%$ das famílias certificadas de ambas as associações.

Para a seleção do representante da família a ser entrevistado foram considerados os seguintes critérios: conhecimento sobre as práticas de manejo do PFNM certificado e a disponibilidade e acessibilidade em contribuir com a pesquisa. As entrevistas foram realizadas individualmente (não sendo permitida a permanência de outro participante) e sempre conduzidas por um único entrevistador, aumentando o grau de independência e uniformidade das avaliações, com questões relacionadas às principais dificuldades enfrentadas pelos manejadores para manter a certificação e as sugestões de mudanças para viabilizar a manutenção do processo. 


\section{RESULTADOS E DISCUSSÃO}

Foi solicitado a cada um dos entrevistados apontar a maior dificuldade encontrada para manter a Certificação Florestal Comunitária em relação aos PFNM's, sendo que a maioria dos manejadores da ASPD elegeram como principais dificuldades a inserção dos produtos no mercado com valor agregado e a falta de informações técnicas.

Para a AMARCA, a maior dificuldade para manter a certificação dos PFNM's está relacionada com a inserção dos produtos no mercado com valor agregado. Dois entrevistados não souberam opinar (TABELA 2).

As diferentes percepções das associações estão relacionadas a diversos aspectos, como a maturidade e a organização de cada uma delas, assim como o relacionamento entre os associados e as instituições de apoio e pesquisa, conforme pôde ser observado durante as entrevistas.

Tabela 2 - Principais dificuldades encontradas pelos Manejadores da ASPD e AMARCA na Certificação de PFNM's, por porcentagem de respostas.

\begin{tabular}{ccc}
\hline Descrição & ASPD (\%) & AMARCA (\%) \\
\hline COMERCIALIZAÇÃO DO PRODUTO & 43 & 60 \\
\hline FALTA DE INFORMAÇÃO A RESPEITO DA CERTIFICAÇÃO & 29 & 0 \\
\hline CONFLITOS ENTRE CERTIFICADOS E NÃO-CERTIFICADOS & 14 & 0 \\
\hline INFRAESTRUTURA PRECÁRIA PARA ESCOAR PRODUTO & 14 & 0 \\
\hline DIFICULDADE EM CUMPRIR NORMAS ESTABELECIDAS PELO FSC & 0 & 20 \\
\hline NÃO ENCONTROU DIFICULDADE & 0 & 20 \\
\hline
\end{tabular}

De uma forma direta ou indireta, outras dificuldades estão relacionadas à comercialização do produto e geração de renda para as famílias de manejadores, uma vez que os conflitos citados estão relacionados ao fato de manejadores não certificados conseguirem o mesmo valor de mercado que os certificados, o que causa desconforto entre os manejadores que seguem as normas estabelecidas pelo FSC. Já a infraestrutura precária poderia ser beneficiada com a implantação de melhorias na logística de escoamento de produtos e/ou com investimentos dos manejadores ou associações provenientes da comercialização do produto.

Considerando que a Certificação Florestal FSC foi criada para incentivar o manejo florestal sustentável dos produtos da floresta e contribuir para que os produtores tenham acesso ao mercado com valor agregado, esta premissa ainda não foi alcançada no que se refere à Certificação Comunitária que inclua os PFNM's nas Associações pesquisadas, pois os produtos certificados continuam sendo vendidos com valor equivalente ao dos produtos não certificado.

Conforme estudo realizado pelo Centro de Trabalhadores da Amazônia (CTA, 2008), iniciativas inovadoras como a certificação florestal comunitária com práticas ambientalmente sustentáveis, que promovem a inclusão social e o desenvolvimento justo e solidário, não têm sido garantia para o estabelecimento de relações comerciais e por isso muitas vezes não são capazes de aumentar a renda do produtor, desestimulando-o manter a certificação.

A prática em optar por produtos certificados ainda é pouco comum em países como o Brasil, onde os consumidores optam pelo melhor preço e/ou produtos com marcas consolidadas no mercado no ato da compra. Segundo pesquisa realizada pelo Instituto Quorum e publicada 
pela ONG Amazônia (2008), estima-se que a cidade de São Paulo, o dos maiores centros urbanos brasileiros, adquira $15 \%$ de tudo o que é retirado da Amazônia. Entretanto, a pesquisa mostra que $70 \%$ dos consumidores paulistanos desistem de comprar produtos certificados se os mesmos itens sem certificação tiverem preço menor e ainda que $47 \%$ dos paulistanos não deixam de consumir um produto, mesmo cientes de que são prejudiciais ao meio ambiente.

Empresas nacionais atuantes em diferentes setores, como extratos naturais, cosméticos, perfumaria e higiene pessoal, caminham para seu estabelecimento no conceito de certificação, visando à inserção efetiva no mercado (FERRO et al., 2006).

A crescente procura pelos PFNM's para a produção de alimentos, fármacos e demais produtos, aliado ao marketing da matéria prima proveniente da floresta amazônica, tem estimulado cada vez mais a extração destes produtos, muitas vezes realizadas por práticas não sustentáveis e por um número cada vez maior de extrativistas. Comunidades tradicionais têm priorizado a coleta destes produtos à manutenção de outras atividades habituais, como a elaboração de do artesanato, caça, pesca e participação de festejos locais. A retirada excessiva destes produtos da floresta pode, em longo prazo, comprometer a regeneração florestal e causar risco de escassez dos produtos, uma vez que estes muitos deles dependem de dispersores da fauna silvestre para a manutenção e disposição destes recursos na floresta.

Neste sentido, a Certificação Florestal se apresenta como uma ferramenta de gestão alternativa em buscar o equilíbrio do sistema, visando minimizar os impactos negativos da exploração florestal e o incentivo ao valor agregado no produto final. Os critérios e indicadores exigidos para o a aprovação do processo considera que a exploração dos PFNM's não devem exceder as taxas acima do normatizado por pesquisas em diversas áreas do conhecimento.

Segundo Shanley et al (2005), a compreensão de fundamentos ecológicos para se estabelecer práticas sustentáveis de extração de PFNM's ainda demanda muito tempo e pesquisa para avaliação de características como tempo de vida, capacidade de brotação, habitat e capacidade de regeneração, entre outros parâmetros.

Desta forma, a falta de informações reclamada pelos manejadores comunitários certificadas muitas vezes não está disponível nem mesmo para os técnicos e pesquisadores, que ainda caminham em direção as respostas demandadas pelos estudos com os não madeireiros.

Todavia, é fato que inúmeros trabalhos de pesquisa estão sendo desenvolvidos com comunidades da Amazônia, o que deve ser considerado quanto as suas consequências aos manejadores. À medida que os pesquisadores se encontram inseridos nas associações buscando informações e coletando dados de campo, cria-se uma expectativa quanto aos resultados obtidos e se as considerações geradas serão agregadas para melhoria do sistema florestal como um todo, porém, segundo os manejadores dessas associações, o retorno dos resultados obtidos é raro, ficando geralmente restritos ao meio acadêmico.

Em complemento às questões levantadas, os manejadores das associações pesquisadas foram questionados a respeito do que poderia ser mudado para melhorar as condições da Certificação Comunitária FSC em relação aos PFNM's, apontando apenas um item mais relevante. Os resultados reforçam os dados já apresentados, onde a inclusão dos produtos com valor agregado no mercado foi a sugestão mais citada (TABELA 3). 
Tabela 3: Sugestões de mudanças no Processo de Certificação pelos Manejadores da ASPD e AMARCA, por porcentagem de respostas.

\begin{tabular}{ccc}
\hline Descrição & ASPD (\%) & AMARCA (\%) \\
\hline APOIO NA COMERCIALIZAÇÃO DO PRODUTO & 57 & 60 \\
\hline INDICADORES COM LINGUAGEM ACESSÍVEL & 14 & 10 \\
\hline AUDITORIA ADAPTADA À COMUNIDADE & 14 & 0 \\
\hline MAIS SEGURANÇA PARA EXTRAIR O PRODUTO & 0 & 10 \\
\hline MELHORAR INFRAESTRUTURA & 0 & 20 \\
\hline NÃO SABE & 15 & 0 \\
\hline
\end{tabular}

Há uma reivindicação marcante por parte das comunidades pesquisadas por melhorias na infraestrutura das unidades familiares de manejo florestal e das comunidades de uma forma geral, como a implantação de sistemas de captação de água e tratamento de resíduos, a adesão de sistema de energia elétrica, as melhorias nos ramais para transporte dos moradores e escoamento de produtos, a implantação de mais escola e postos de saúde de melhor qualidade, entre outras.

Estas reivindicações condizem com o plano idealizado pelo Conselho de Manejo Florestal (FSC) para a Certificação Florestal Comunitária, o de valorizar os moradores rurais da Amazônica em busca da sustentabilidade de exploração dos recursos naturais, levando em consideração os aspectos ambientais, como também os sociais e econômicos.

Em relação à linguagem acessível e auditoria adaptada à comunidade, os manejadores afirmam que muitas vezes não compreendem o que é solicitado e desta maneira acabam descumprindo a normatização estabelecida para a certificação. O mesmo ocorre com a reivindicação por mais segurança.

Já a questão de mercado foi apontada, por ambas as associações, como a mudança mais relevante necessária a manutenção do processo de certificação. Considerando que a certificação florestal demanda mais trabalho e custo ao produtor, a vantagem a esta prática deve ser financeira, embora o objetivo da certificação também contemple benefícios sociais e ambientais.

Vale ressaltar que muitos manejadores não possuem a competência necessária a inserção de seus produtos no mercado sem assessoria de instituições de apoios especializadas.

Desta forma, se faz necessário um acompanhamento pós-certificação e a capacitação dos manejadores para questões relativas à comercialização de seus produtos.

\section{CONCLUSÃO}

A avaliação da percepção dos manejadores certificados consiste em uma importante ferramenta de adaptações e aperfeiçoamento das condições existentes para a comercialização de seus produtos, considerando as dificuldades e expectativas dos moradores tradicionais.

A comercialização dos produtos florestais não madeireiros certificados continua sendo um ponto crítico dentro do processo, sendo que a inserção destes produtos no mercado com valor agregado é a maior dificuldade encontrada pelos manejadores e também é considerada a reivindicação mais relevante pelas duas associações pesquisadas. 
Visando a viabilidade do processo de certificação e seus benefícios em relação ao uso sustentável dos produtos da floresta, deve haver um acompanhamento pós-certificação com programas de capacitação de agentes comunitários na comercialização de seus produtos, assim como a adoção de práticas como a organização em cooperativas agrícolas.

Neste último caso, além de contribuir com a organização e gestão do processo de certificação, o cooperativismo pode reduzir os custos inerentes aos procedimentos e auditorias, tornando os produtos extraídos da floresta mais competitivos no mercado.

Por fim, a adoção de uma estratégia de comunicação e difusão de informações sobre certificação florestal e seus benefícios se faz necessário não somente aos manejadores, mas também ao mercado consumidor, para que haja um estímulo à aquisição de produtos que atendam critérios específicos em relação aos propósitos estabelecidos.

\section{AGRADECIMENTOS}

Agradecemos a todos os Manejadores das Associações dos Seringueiros Porto Dias e dos Moradores e Agroextrativistas do Remanso de Capixaba pelas informações e disponibilidade em participar desta pesquisa, a Maria das Graças Carlos da Silva e Juliete Nathália Pereira pelas sugestões e revisões, à Embrapa Acre por meio do projeto Kamukaia, CTA, SEF e UNIR pelo apoio logístico.

\section{REFERÊNCIAS BIBLIOGRÁFICAS}

1. ALCORN, J. B. Process as Resource: The Traditional Agricultural Ideology of Bora and Huastec Resource Management and Implications for Research. Advances in Economic Botany, v. 7, p. 63-77, 1989.

2. AMARAL, P.\& AMARAL NETO, M. Manejo Florestal Comunitário: processos e aprendizagens na Amazônia brasileira e na América Latina. Belém: IEB e IMAZON, 2005.

3. AMAZÔNIA. Preço alto ainda é empecilho para desenvolvimento de produtos certificados 27/10/2008. Disponível em: < www.amazonia.org.br >. Acesso em: 07 nov. 2012.

4. Brechin,S.R.; Wilshusen, P.; Fortwangler,C. and WestBRECHIN, P. Beyond the square wheel: Toward a more comprehensive understanding of biodiversity conservation as social and political process. Society and Natural Resources, v. 15, p. 41-64. 2002.

5. CTA. Centro de Trabalhadores da Amazônia. Apoio ao comercio justo e solidário de óleo de copaíba manejado e certificado: uma estratégia de geração de renda para pequenos produtores (as) florestais e de Conservação Ambiental no Estado do Acre. Relatório Técnico. Acervo do CTA. 2008.

6. ESCOBAL, J. \& ALDANA, U. Are nontimber forest products the antidote to rainforest degradation? Brazil nut extraction in Madre de Dios, Peru. World Development, v. 31, N. 11, p. 1873-1887, 2003..

7. FARNSWORTH,N.R.; AKERELE O.; BINGEL, A.S.; SOEJARTO, D.D. and GUO Z. Medicinal plants in therapy. Bulletin of the World Health Organization, v. 63, p. 965-981. 1985.

8. FERRO, A. F. P.; BONACELLI, M. B. M. and ASSAD, A. L. D. Oportunidades tecnológicas e 
estratégias concorrenciais de gestão ambiental: o uso sustentável da biodiversidade brasileira. Rev. Gestão \& Produção, v. 13, n. 3, p. 489-501, set.-dez. 2006.

9. HEYWOOD, V. H. \& IRIONDO, J. M. Plant Conservation: old problems, news perspectives. Biological Conservation, v. 113, p. 321-335, 2003.

10. HIGMAN, S.\& NUSSBAUM, R. How standards constrain the certification of small forest enterprises. Report for UK DFID Forestry Research Program 2002.

11. HUMPHRIES, S. \& KAINER, K. Local Perception of Forest Certification for Community-based enterprises. Forest Ecology and Management, v. 235, p. 30-43, 2006.

12. JONES, E. C. McLAIN, R.J. and WEIGGAND,J. Nontimber forest products in the United States. Lawrence, Kansas: University Press of Kansas, 2002.

13. LIMA, A. C. B.; SPAROVEK, G. E certificar, faz diferença? Estudo de avaliação de impacto da certificação FSC/RAS. Piracicaba: Imaflora, 2009. 96 p.

14. LIMA, D. \& POZZOBON, J. Amazônia Socioambiental. Sustentabilidade Ecológica e Diversidade Social. Dossiê Amazônia Brasileira II. Rev. Estudos Avançados, v. 19, n. 54. São Paulo, 2005.

15. MOLNAR, A.; SCHERR, S.J. and KHARE, A. Strategies to Protect Forests and Respect Rights. Washington D.C.: Forest Trends and Ecoagriculture. Parners, 2004.

16. MYERS, G.P., NEWTON, A. AND MELGAREJO, O. The influence of canopy gap size on natural regeneration of Brazil nut (Bertholletia excelsa) in Bolivia. Forest Ecol. Manag, v. 127, p. 119$128,2000 . N$

17. NEPSTAD D, MCGRATH D, ALENCAR A, BARROS AC, CARVALHO G, SANTILLI M, DIAZ V. and MDEL C. Frontier Governance in Amazonia. Science, n. 295, 2002, p. 629-631.

18. PIERCE, A. R. \& LAIRD, S. A. In search of comprehensive standards for non-timber forest products in the botanicals trade. International Forestry Review, v. 5(2), p. 138-147, 2003.

19. PINTO, L.F.G.; SHANLEY, P. and COTA GOMES, A.P. Experience with NTFP Certification in Brazil. Forest, Trees and Livelihoods, v. 18, p. 37-54, 2008.

20. QUEVEDO, L. Forest certification in Bolivia. In: CASHORE, B. et al. (Eds.). Confronting Sustainability: Forest Certification in Developing and Transitioning Countries. New Haven, CT: Yale School of Forestry and Environmental Studies Press, 2006.

21. ROBINSON, D.\& BROWN, L. The SLIMFs Initiative: A Progress Report, FSC. 2002.

22. SHANLEY, P. et al. Além da Madeira: A Certificação de Produtos Florestais não-madeireiros. Bogor, Indonésia: Centro de Pesquisa Florestal Internacional CIFOR, 2005. 153p.

23. SILVA, M. A. F. Métodos e Técnicas de Pesquisa. 2. ed. Curitiba: Impex, 2005, p. 110-113.

24. SMARTWOOD \& IMAFLORA. Resumo Público de Certificação FSC Programa Smartwood da Associação dos Seringueiros da Reserva Extrativista São Luiz do Remanso - ASSER. Capixaba, 2006. 56 p.

25. Resumo Público de Re - Certificação FSC Programa Smartwood da Associação Seringueira Porto Dias - ASPD. Acrelândia, 2005. 16 p.

26. THORNBER, K. \& MARKOPOULOS, M. Certification: Its Impacts and Prospects for Community Forests, Stakeholders, and Markets. Londres: International Institute for Environment and 
Development (IIED), 2001.

27. WADT, L. H. de; KEINER, K. A. and GOMES-SILVA, D. A. P. Estrutura populacional e produtividade de Bertholletia excelsa H.B.K. no sudoeste da Amazônia. In: CONGRESSO DE ECOLOGIA DO BRASIL, 6, 2003, Fortaleza-CE. Anais de trabalhos completos. Fortaleza: Universidade Federal do Ceará, p. 89-90. 2003.

28. WADT, L. H. O.; KAINER, K.A.; STAUDHAMMERC.L. and SERRANO, R.O.P. Sustainable forest use in Brazilian extractive reserve: Natural regeneration of Brazil nut exploited populations. Biological Conservation, v. 141, p. 332-346, 2008.

29. WENBAN-SMITH, M., W, NUSSBAUM R., GARFORTH M and SCRASE H. An analysis of the barriers faced by small scale farmers and communities producing timber outside a conventional forest matrix and recommendations for progress. Oxford: Proforest. 2001. 